\title{
A Bivariate Generalized Exponential Model for the Effect of Endocrine Responses in Human with Postprandial Distress Syndrome
}

\author{
S.Lakshmi* and N.Durgadevi** \\ *Principal, Government Arts and Science College, Peravurani - 614 804, Thanjavur - Dt (TN), \\ **Research Scholar, P.G and Research Department of Mathematics, K.N.Govt. Arts College for Women, \\ Thanjavur - Dt $(T N)$,
}

\begin{abstract}
Suppose a system has two components. Each component is subject to individual independent stress say $U_{1}$ and $U_{2}$ respectively. The system has an overall stress $U_{3}$ which has been transmitted to both the components equally, independent of their individual stresses. Therefore, the observed stress at the two components are $X_{1}=\max \left\{U_{1}, U_{3}\right\}$ and $X_{2}=\max \left\{U_{2}, U_{3}\right\}$ respectively. If it is assumed that each component has been maintained independently and also there is an overall maintenance. Due to component maintenance, suppose the lifetime of the individual is increased by $U_{i}$ amount and because of the overall maintenance, the lifetime of each component is increased by $U_{3}$ amount. The study in the Application part is fully characterized the neuroendocrine pathways involved in the gastric response to mental stress in patients with PDS. The increased severity of dyspeptic symptoms induced by acute mental stress in patients with PDS is associated with an enhanced sympathetic output and higher serum levels of stress hormones, ACTH, and cortisol. If $\left(X_{1}, X_{2}\right) \sim B V G E\left(\alpha_{1}, \alpha_{2}, \alpha_{3}\right)$, then the joint PDF of $\left(X_{1}, X_{2}\right)$ for $x_{1}>0, x_{2}>0$, is obtained for the above two variables.
\end{abstract}

Keywords: HPA axis, LF/HF ratio, mental stress, postprandial distress syndrome, CRH, ACTH, cortisol. 2010 AMS Classification: 62HXX, 60EXX.

\section{MATHEMATICAL MODEL:}

Bivariate generalized exponential distribution:

The univariate GE distribution has the following cumulative distribution function(CDF) and probability density function(PDF) respectively for $x>0$;

$$
F_{G E}(x ; \alpha, \lambda)=\left(1-e^{-\lambda x}\right)^{\alpha}, f_{G E}(x ; \alpha, \lambda)=\alpha \lambda e^{-\lambda x}\left(1-e^{-\lambda x}\right)^{\alpha-1}
$$

Here $\alpha>0$ and $\lambda>0$ are the shape and scale parameters respectively. It is clear that for $\alpha=1$, it coincides with the exponential distribution [1]. From now on a GE distribution with the shape parameter $\alpha$ and the scale parameter $\lambda$ will be denoted by $\operatorname{GE}(\alpha, \lambda)$. For brevity when $\lambda=1$, we will denote it by $G E(\alpha)$ and for $\alpha=1$, it will be denoted by $\operatorname{Exp}(\lambda)$ [5]. From now on unless otherwise mentioned, it is assumed that $\alpha_{1}>0, \alpha_{2}>0, \alpha_{3}>0, \lambda>0$. Suppose $U_{1} \sim G E\left(\alpha_{1}, \lambda\right), U_{1} \sim G E\left(\alpha_{2}, \lambda\right)$ and $U_{3} \sim \operatorname{GE}\left(\alpha_{3}, \lambda\right)$ and they are mutually independent. Here' $\sim$ 'means follows or has the distribution . Now define $X_{1}=$ $\max \left\{U_{1}, U_{3}\right\}$ and $X_{2}=\max \left\{U_{2}, U_{3}\right\}$. Then we say that the bivariate vector $\left(X_{1}, X_{2}\right)$ has a bivariate generalized exponential distribution with the shape parameters $\alpha_{1}, \alpha_{2}$ and $\alpha_{3}$ and scale parameter $\lambda[6]$. We will denote it by $\operatorname{BVGE}\left(\alpha_{1}, \alpha_{2}, \alpha_{3}, \lambda\right)$. Now for the rest of the discussions for brevity, we assume that $\lambda=1$, although the results are true for general $\lambda$ also. The BVGE distribution with $\lambda=1$ will be denoted by $\operatorname{BVGE}\left(\alpha_{1}, \alpha_{2}, \alpha_{3}\right)$. Before providing the joint $\mathrm{CDF}$ or $\mathrm{PDF}$, we first mention how it may occur in practice $[7,9]$.

\subsection{STRESS MODEL:}

Suppose a system has two components. Each component is subject to individual independent stress say $U_{1}$ and $U_{2}$ respectively. The system has an overall stress $U_{3}$ which has been transmitted to both the components equally, independent of their individual stresses. Therefore, the observed stress at the two components are

$X_{1}=\max \left\{U_{1}, U_{3}\right\}$ and $X_{2}=\max \left\{U_{2}, U_{3}\right\}$ respectively.

\subsection{MAINTENANCE MODEL:}

Suppose a system has two components and it is assumed that each component has been maintained independently and also there is an overall maintenance. Due to component maintenance, 
suppose the lifetime of the individual is increased by $U_{i}$ amount and because of the overall maintenance, the lifetime of each component is increased by $U_{3}$ amount. Therefore, the increased lifetimes of the two components are $X_{1}=\max \left\{U_{1}, U_{3}\right\}$ and $X_{2}=\max \left\{U_{2}, U_{3}\right\}$ respectively.

The following results will provide the joint CDF, joint PDF and conditional PDF.

Theorem1.1:

If $\left(X_{1}, X_{2}\right) \sim B V G E\left(\alpha_{1}, \alpha_{2}, \alpha_{3}\right)$, then the joint $\mathrm{CDF}$ of $\left(X_{1}, X_{2}\right)$ for $x_{1}>0, x_{2}>0$, is $F_{X_{1, X}}\left(x_{1}, x_{2}\right)=$ $\left(1-e^{-x_{1}}\right)^{\alpha_{1}}\left(1-e^{x_{2}}\right)^{\alpha_{2}}\left(1-e^{-Z}\right)^{\alpha_{3}}$,

where $z=\min \left\{x_{1}, x_{2}\right\}$.

Corollary 1.1:

The joint $\mathrm{CDF}$ of the $\operatorname{BVGE}\left(\alpha_{1}, \alpha_{2}, \alpha_{3}\right)$, can also be written as

$$
\begin{gathered}
F_{X_{1}, X_{2}}\left(x_{1}, x_{2}\right)=F_{G E}\left(x_{1} ; \alpha_{1}\right) F_{G E}\left(x_{2}, \alpha_{2}\right) F_{G E}\left(z ; \alpha_{3}\right) \\
\quad=F_{G E}\left(x_{1} ; \alpha_{1}+\alpha_{3}\right) F_{G E}\left(x_{2}, \alpha_{2}\right) \text { if } x_{1}<x_{2} \\
\quad=F_{G E}\left(x_{1} ; \alpha_{1}\right) F_{G E}\left(x_{2}, \alpha_{2}+\alpha_{3}\right) \text { if } x_{2}<x_{1} \\
\quad=F_{G E}\left(x ; \alpha_{1}+\alpha_{2}+\alpha_{3}\right) \text { if } x_{1}=x_{2}=x .
\end{gathered}
$$

Theorem 1.2:

If $\left(X_{1}, X_{2}\right) \sim B V G E\left(\alpha_{1}, \alpha_{2}, \alpha_{3}\right)$, then the joint PDF of $\left(X_{1}, X_{2}\right)$ for $x_{1}>0, x_{2}>0$, is

$f_{X_{1}, X_{2}}\left(x_{1}, x_{2}\right)=\left\{\begin{array}{ccc}f_{1}\left(x_{1}, x_{2}\right) & \text { if } & 0<x_{1}<x_{2}<\infty \\ f_{2}\left(x_{1}, x_{2}\right) & \text { if } & 0<x_{2}<x_{1}<\infty \\ f_{0}(x) & \text { if } & 0<x_{1}=x_{2}<\infty,\end{array}\right.$

Where

$$
\begin{aligned}
f_{1}\left(x_{1}, x_{2}\right) & =f_{G E}\left(x_{1} ; \alpha_{1}+\alpha_{3}\right) f_{G E}\left(x_{2} ; \alpha_{2}\right) \\
& =\left(\alpha_{1}+\alpha_{3}\right) \alpha_{2}\left(1-e^{-x_{1}}\right)^{\alpha_{1}+\alpha_{3}-1}\left(1-e^{-x_{2}}\right)^{\alpha_{2}-1} e^{-x_{1}-x_{2}} \\
f_{2}\left(x_{1}, x_{2}\right) & =f_{G E}\left(x_{1} ; \alpha_{1}\right) f_{G E}\left(x_{2} ; \alpha_{2}+\alpha_{3}\right) \\
& =\left(\alpha_{2}+\alpha_{3}\right) \alpha_{1}\left(1-e^{-x_{1}}\right)^{\alpha_{1}-1}\left(1-e^{-x_{2}}\right)^{\alpha_{2}+\alpha_{3}-1} e^{x_{1}-x_{2}} \\
f_{0}(x) \quad & =\frac{\alpha_{3}}{\alpha_{1}+\alpha_{2}+\alpha_{3}} f_{G E}\left(x ; \alpha_{1}+\alpha_{2}+\alpha_{3}\right) \\
& =\alpha_{3}\left(1-e^{-x}\right)^{\alpha_{1}+\alpha_{2}+\alpha_{3}-1} e^{-x} .
\end{aligned}
$$

Proof :

The expressions for $f_{1}(.,$.$) and f_{2}(.,$.$) can be obtained simply by taking \frac{\partial^{2} F_{X_{1}, X_{2}\left(x_{1}, x_{2}\right)}}{\partial x_{1} \partial x_{2}}$ for $x_{1}<x_{2}$ and $x_{2}<x_{1}$ respectively But $f_{0}($.$) cannot be obtained in the same way. Using the facts that$ $\int_{0}^{\infty} \int_{0}^{x_{2}} f_{1}\left(x_{1}, x_{2}\right) d x_{1} d x_{2}+\int_{0}^{\infty} \int_{0}^{x_{1}} f_{2}\left(x_{1}, x_{2}\right) d x_{2} d x_{1}+\int_{0}^{\infty} f_{0}(x) d x=1$,

$\int_{0}^{\infty} \int_{0}^{x_{2}} f_{1}\left(x_{1}, x_{2}\right) d x_{1} d x_{2}=\alpha_{2} \int_{0}^{\infty}\left(1-e^{-x}\right)^{\alpha_{1}+\alpha_{2}+\alpha_{3}-1} e^{-x} d x$

$\int_{0}^{\infty} \int_{0}^{x_{1}} f_{2}\left(x_{1}, x_{2}\right) d x_{2} d x_{1}=\alpha_{1} \int_{0}^{\infty}\left(1-e^{-x}\right)^{\alpha_{1}+\alpha_{2}+\alpha_{3}-1} e^{-x} d x$

note that $\int_{0}^{\infty} f_{0}(x) d x=\alpha_{3} \int_{0}^{\infty}\left(1-e^{-x}\right)^{\alpha_{1}+\alpha_{2}+\alpha_{3}-1} e^{-x} d x=\frac{\alpha_{3}}{\alpha_{1}+\alpha_{2}+\alpha_{3}}$.

Therefore, the result follows.

\section{APPLICATION}

Mental stress may alter gastric sensory motor function. The aim of the study is to assess postprandial autonomic nervous system activity and stress hormones in response to acute mental stress in dyspeptic patients.

Functional dyspepsia (FD) is a highly prevalent gastro-intestinal disorder characterized by symptoms originating from the gastroduodenal region, in the absence of underlying organic diseases that would readily explain the symptoms. Based on the distinction between meal-induced and mealunrelated symptoms, FD is subdivided into two diagnostic categories (i) Postprandial distress syndrome (PDS), characterized by postprandial fullness and early satiation, and (ii) epigastric pain syndrome (EPS) characterized by epigastric pain and burning.[2]

Studies concerning the relationship between stressful life events and FD have yielded conflicting results. Some studies have shown that subjects with FD experienced more stressful life events than healthy controls whereas other studies have failed to find any significant differences.[4]

The aim of this study is to thoroughly investigate every step of this intricate link by evaluating the serum concentrations of stress hormones, the autonomous activity of the nervous system (ANS), and the gastric sensorimotor function, in response to an experimentally induced 
acute mental stress in patients with PDS [8]. The study is designed to test our hypothesis that in this subset of patients, the modulation of gastric function under stressful conditions is related to HPA axis and ANS activity.

We have corrected individuals $\mathrm{LF} / \mathrm{HF}$ ratio values for the anxiety scores in each subject and performed a comparison between basal and stress conditions, without finding any statistical significance. In patients, a significantly higher ACTH level was found in stress condition, whereas in healthy subjects no modifications was recorded. Patients showed a significantly higher level in stress condition, whereas in healthy subjects cortisol levels in both conditions were similar.

The lack of significant differences between LF/HF ratio values corrected for anxiety score between basal and stress conditions in patients confirms that the higher level of underlying anxiety in patients represents an important factor to determine the enhanced neuroendocrine response to stress.

The study here is to fully characterize the neuroendocrine pathways involved in the gastric response to mental stress in patients with PDS. The increased severity of dyspeptic symptoms induced by acute mental stress in patients with PDS is associated with an enhanced sympathetic output and higher serum levels of stress hormones, ACTH, and cortisol.

In conclusion, our findings contribute to shed light on complex link between the central nervous system and the gastrointestinal tract, and specifically to help to clarify the mechanisms that underlie functional gut modifications induced by mental stress in FD.

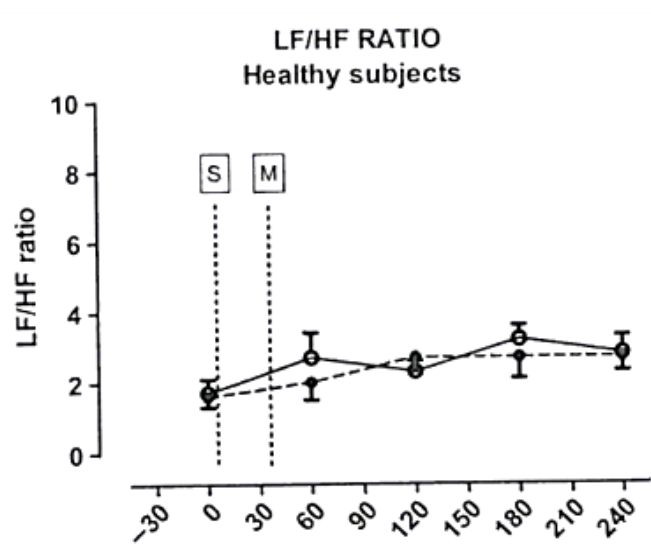

Fig2.1

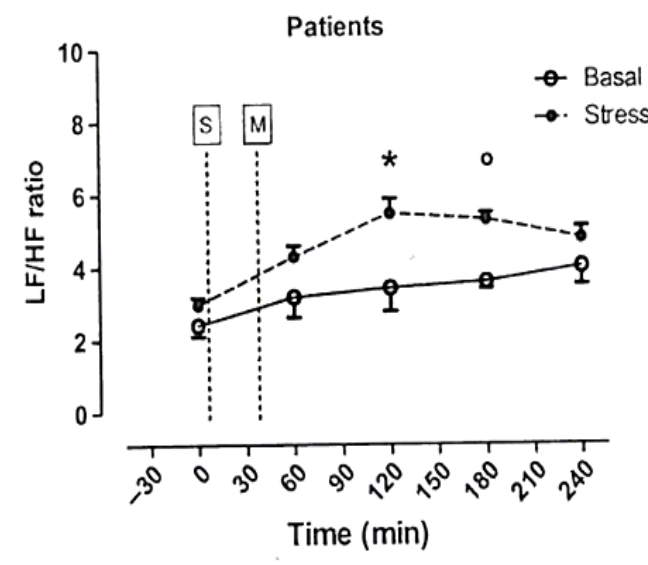

Fig2.2

Low/high frequency component ( $L F / H F)$ ratio in patients and controls during basal and stress sessions of this study. In patients, mental stress induces a prolonged increase of $L F / H F$ ratio in the postprandial period, interpreted as an increase in sympathetic output.In controls, no differences were found between basal and stress condition. Each plotted value on the graph expresses the LF/HF ratio of the previous $60 \mathrm{~min}$. S,stress; $M$ meal. $* P<0.01$ and $\circ P<0.05$ vs basal.

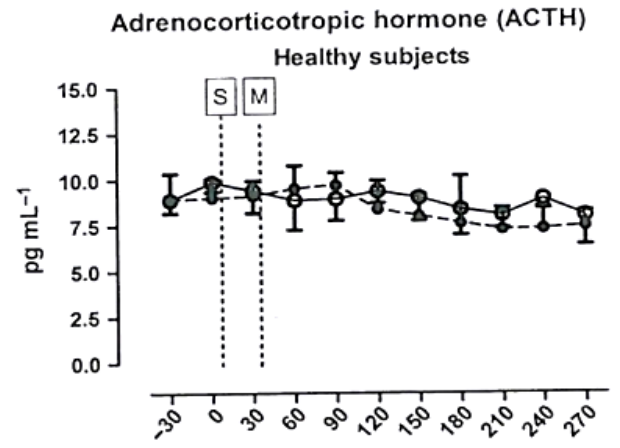

Fig 2.3

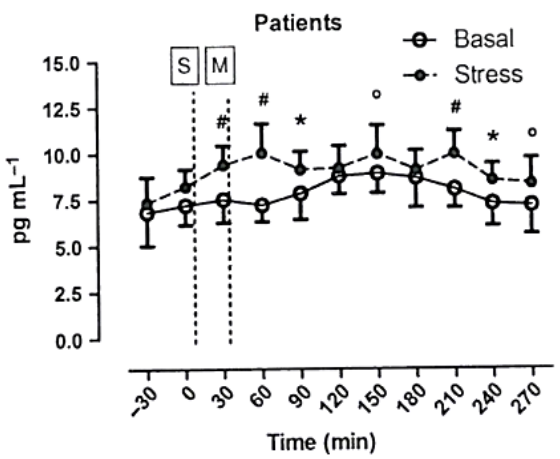

Fig 2.4

Adrenocorticotropic hormone (ACTH) serum levels in healthy subjects and patients. In patients, a significantly higher ACTH level was found in stress condition, whereas in healthy subjects no modifications were recorded. $S$, stress; $M$, meal.\# $P<0.001, * P<0.01$, and o $P<0.05$ vs basal. 


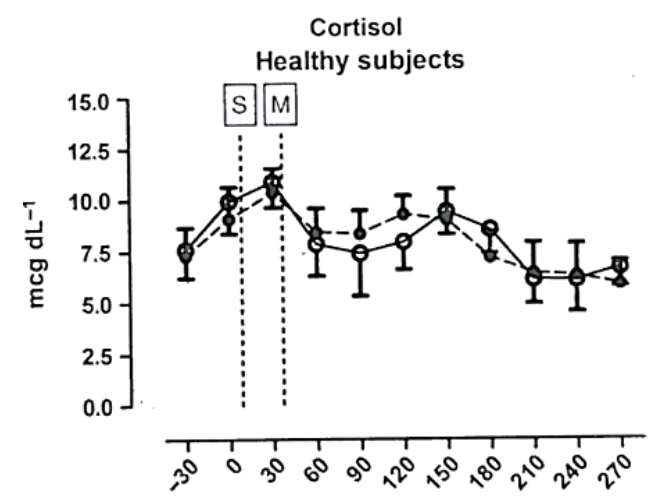

Fig 2.5

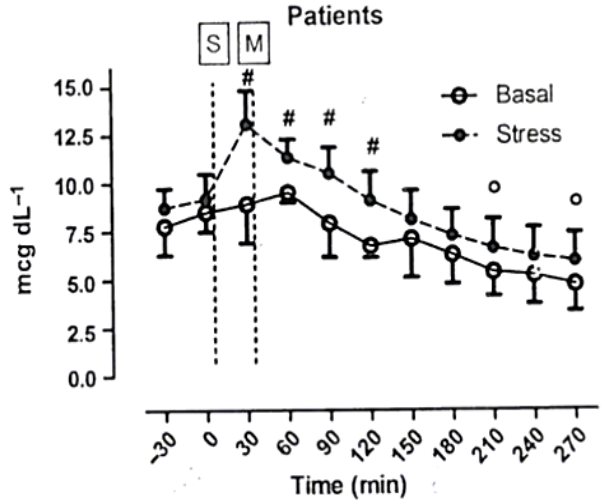

Fig2.6

Cortisol serum levels in healthy subjects and patients. Patients showed a significantly higher level in stress condition, whereas in healthy subjects cortisol level in both conditions were similar. S, stress; $M$, meal. $\# P<0.001$ and $0 P<0.05$ basal.

\section{MATHEMATICAL RESULTS}

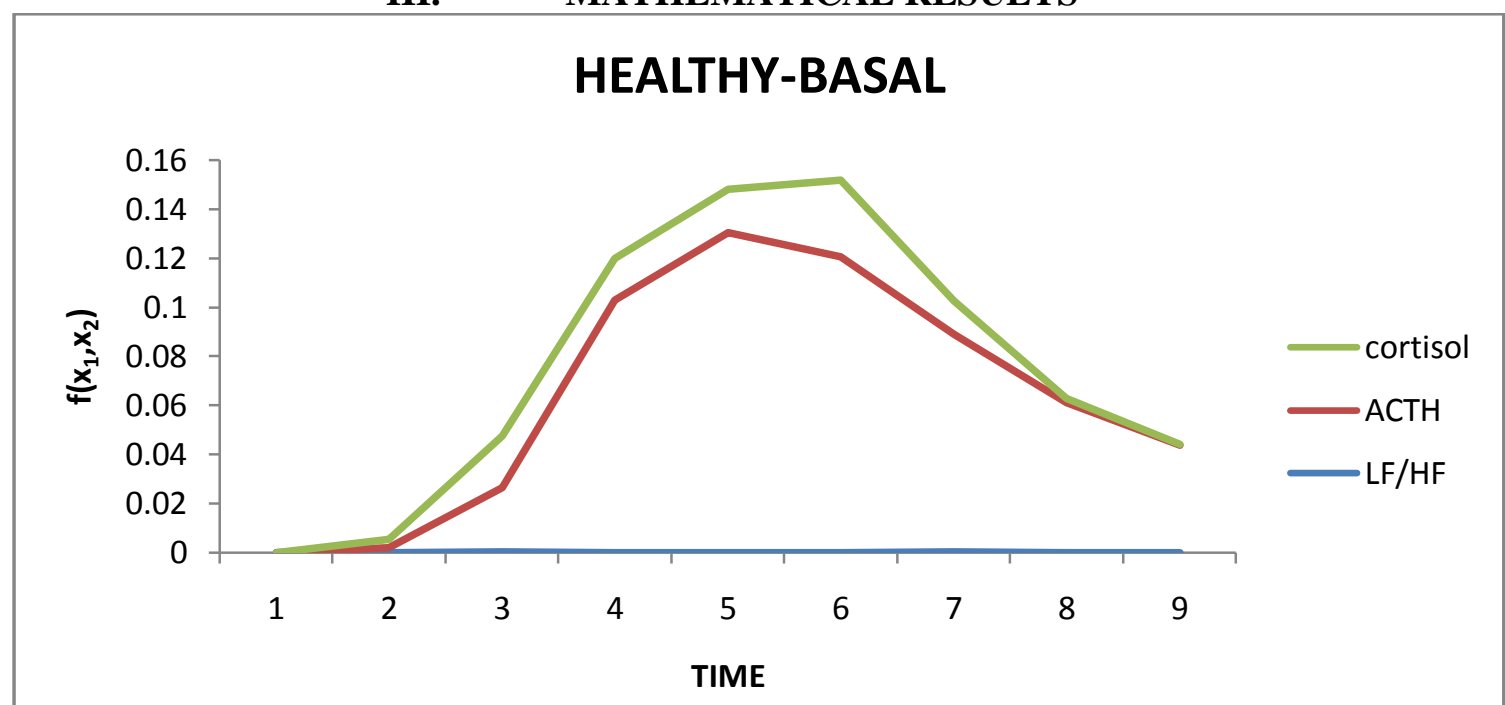

Fig3.1

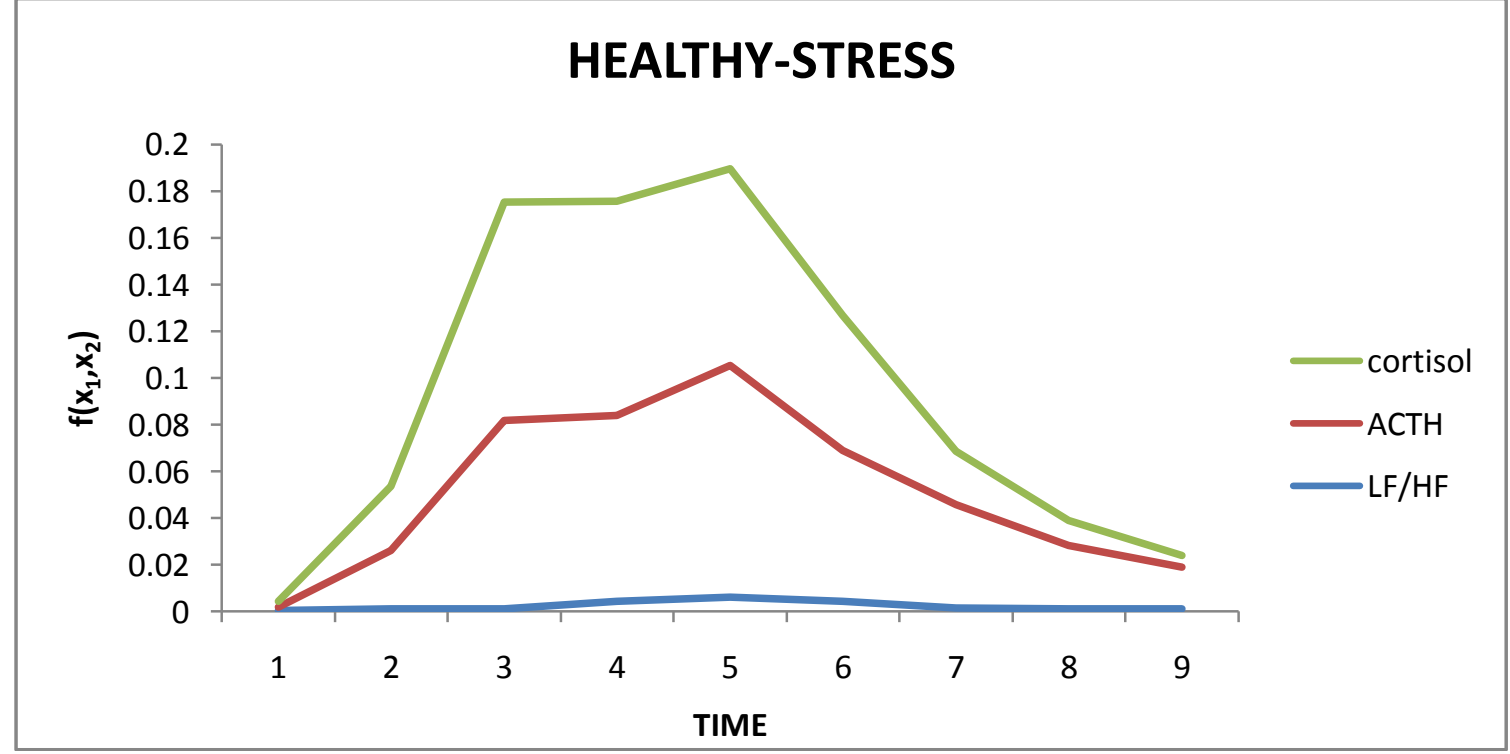

Fig3.2 


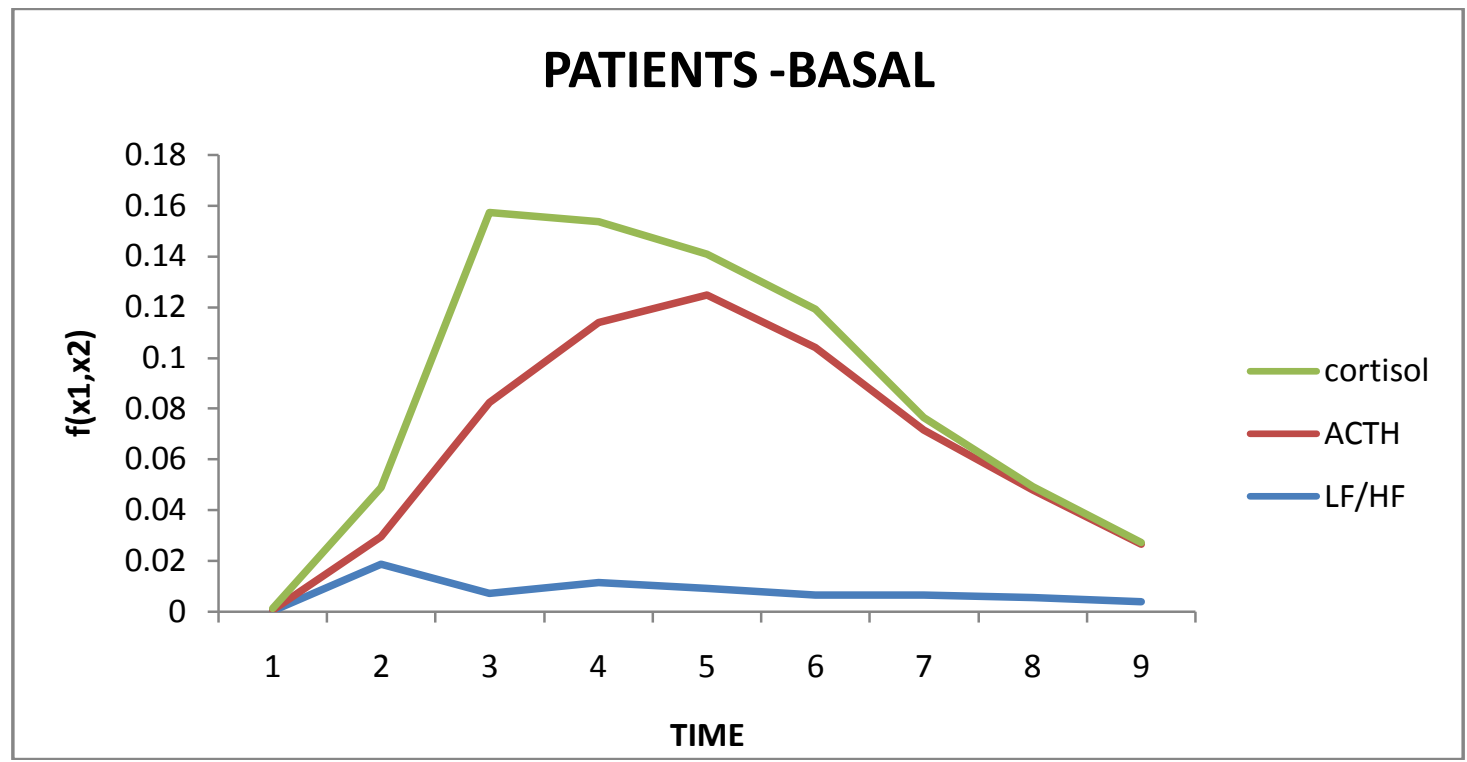

Fig3.3

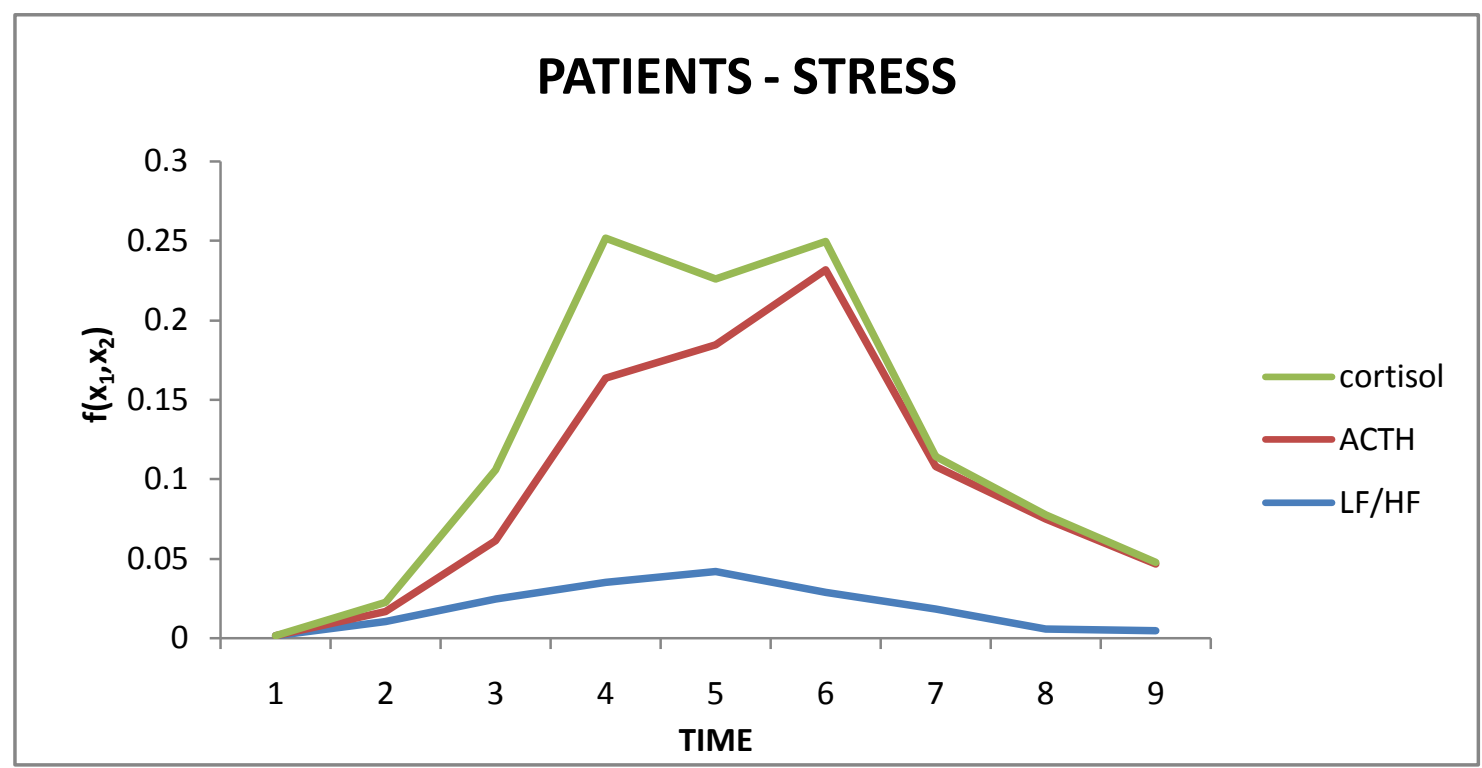

Fig3.4

\section{CONCLUSION}

Suppose a system has two components. Each component is subject to individual independent stress say $U_{1}$ and $U_{2}$ respectively. The system has an overall stress $U_{3}$ which has been transmitted to both the components equally, independent of their individual stresses. Therefore, the observed stress at the two components are $X_{1}=\max \left\{U_{1}, U_{3}\right\}$ and $X_{2}=\max \left\{U_{2}, U_{3}\right\}$ respectively. If it is assumed that each component has been maintained independently and also there is an overall maintenance. Due to component maintenance, suppose the lifetime of the individual is increased by $U_{i}$ amount and because of the overall maintenance, the lifetime of each component is increased by $U_{3}$ amount. The study in the Application part is fully characterized the neuroendocrine pathways involved in the gastric response to mental stress in patients with PDS. The increased severity of dyspeptic symptoms induced by acute mental stress in patients with PDS is associated with an enhanced sympathetic output and higher serum levels of stress hormones, ACTH, and cortisol. If $\left(X_{1}, X_{2}\right) \sim B V G E\left(\alpha_{1}, \alpha_{2}, \alpha_{3}\right)$, then the joint PDF of $\left(X_{1}, X_{2}\right)$ for $x_{1}>0, x_{2}>0$, is obtained for the above two variables which are well explained in the figures 3.1, 3.2, 3.3, 3.4 of the Mathematical Results. There is no change in the level of cortisol and ACTH between the groups of healthy subjects and patients in basal level. But, there is a vast change in the cortisol and ACTH level between the groups of healthy stress and the patients stress. There is no major change in LF/HF for all the four cases. 


\section{REFERENCES}

[1] Arnold, B., (1967) “A note on multivariate distributions with specified marginals", Journal of the American Statistical Association, Vol 62, pp 1460-1461 .

[2] Aro P, Talley NJ., Ronkainen J et al. (2009) Anxiety is associated with Swedish populationbased study. Gastroenterology, Vol 137, pp 94-100.

[3] Bremner JD., Bolus R., Mayer EA., (2007) Psychometric properties of the Early Trauma Inventory -Self Report: J Nerv Ment Dis, Vol 195, pp 211- 218.

[4] Chang L., Sundaresh S., Elliott J., (2009) Dysregulation of the hypothalamic pituitary-adrenal (HPA)axis in irritable bowel syndrome: Neurogastroenterol Motil, Vol 21, pp 149-159.

[5] Gupta R D., and Kundu D., (1999) "Generalized exponential distributions", Australian and New Zealand Journal of statistics, Vol 41, pp 173-188.

[6] Gupta R D., and Kundu D.,(2007) "Generalized exponential distributions: existing results and some recent developments" ,Journal of Statistical and Inference, Vol 137, pp 3525-3526.

[7] Marshall A W., and Olkin I., (1967) "A multivariate exponential distribution", Journal of the American Statistical Association, Vol 6, pp 30-44.

[8] Ott V., Fredrich M., Prilop S et al. (2011) Food anticipation and subsequent food withdrawal increase serum cortisol in men: Physiol Behav, Vol 103, pp 594-599.

[9] Sarhan A., and Balakrishnan., (2007) " A new class of bivariate distribution and its mixure", Journal of the Multivariate Analysis, Vol 98, pp 508-1527.

[10] Stengel A., Tache Y., (2009) Neuroendocrine control of the gut during stress: corticotrophin releasing factor signalling pathways in the spotlight. Annu Rev Physiol, Vol 71, pp 219-239.

[11] Van den Elzen BD., Van den Wijngaard RM., Tytgat GN et al. (2007) Influence of corticotropin -releasing hormone on gastric sensitivity and motor function in healthy volunteers. Eur J Gastro enterol Hepatol Vol 19, pp 401-407. 